\title{
The topology of the permutation pattern poset
}

\author{
Peter R. W. McNamara ${ }^{1 \mid k}$ and Einar Steingrímsson $\|^{2}$ \\ ${ }^{1}$ Department of Mathematics, Bucknell University, Lewisburg, PA 17837, USA \\ ${ }^{2}$ Department of Computer and Information Sciences, University of Strathclyde, Glasgow G1 1XH, UK
}

\begin{abstract}
The set of all permutations, ordered by pattern containment, forms a poset. This extended abstract presents the first explicit major results on the topology of intervals in this poset. We show that almost all (open) intervals in this poset have a disconnected subinterval and are thus not shellable. Nevertheless, there seem to be large classes of intervals that are shellable and thus have the homotopy type of a wedge of spheres. We prove this to be the case for all intervals of layered permutations that have no disconnected subintervals of rank 3 or more. We also characterize in a simple way those intervals of layered permutations that are disconnected. These results carry over to the poset of generalized subword order when the ordering on the underlying alphabet is a rooted forest. We conjecture that the same applies to intervals of separable permutations, that is, that such an interval is shellable if and only if it has no disconnected subinterval of rank 3 or more. We also present a simplified version of the recursive formula for the Möbius function of decomposable permutations given by Burstein et al.

Résumé. L'ensemble de toutes les permutations, ordonné par l'endiguement des motifs, forme un ensemble partiellement ordonné. Ce résumé éxtensif présente les premiers résultats majeurs explicites sur la topologie des intervalles dans ce poset. Nous montrons que presque tous les intervalles (ouverts) dans ce poset ont un sous-intervalle déconnecté et donc ne sont pas enveloppable ("shellable"). Néanmoins, il semble y avoir de grandes classes d'intervalles qui sont enveloppable et ont donc le type homotopique d'un bouquet de sphères. Nous démontrons que ce soit le cas pour tous les intervalles de permutations étagés qui n'ont pas de sous-intervalles disjoints de rang 3 ou plus. Nous caractérisons également de manière simple les intervalles de permutations étagés qui sont déconnectés. Ces résultats portent sur l'ensemble partiellement ordonné de l'ordre de sous-mot généralisé lorsque l'ordre sur l'alphabet sous-jacent est une forêt enracinée. Nous faisons la conjecture que la même chose s'applique à des intervalles de permutations séparables, c'est à dire, un tel intervalle est enveloppable si et seulement s'il n'a pas de sous-intervalle déconnecté de rang 3 ou plus. Nous présentons également une version simplifiée de la formule récursive pour la fonction de Möbius de permutations décomposables proposés par Burstein et al.
\end{abstract}

Keywords: pattern poset, shellable, disconnected, layered permutations, generalized subword order, Möbius function

\section{Introduction}

An occurrence of a pattern $p$ in a permutation $\pi$ is a subsequence of $\pi$ whose letters appear in the same relative order of size as those in $p$. For example, the permutation 416325 contains two occurrences of the

*Email: peter. mcnamara@bucknell. edu. Partially supported by grant \#245597 from the Simons Foundation.

$\dagger$ Email: einar@alum.mit. edu. Supported by grant no. 090038013 from the Icelandic Research Fund. 
pattern 231, in 463 and 462 . The origin of the study of permutation patterns can be traced back a long way. In the 1960s and 70s the number of permutations of length $n$ avoiding (having no occurrence of) any one of the six patterns of length 3 was determined by Knuth [Knu75, Exercise 2.2.1.5] and Rogers [Rog78]. In all of these cases, which are easily seen to fall into two equivalence classes, the numbers in question turn out to be the $n$-th Catalan number. In a seminal 1985 paper, Simion and Schmidt [SS85] then did the first systematic study of pattern avoidance, and established, among other things, the number of permutations avoiding any given set of patterns of length 3 . In the last two decades this research area has grown steadily, and explosively in recent years, with several different directions emerging. For a recent comprehensive survey see [Kit11], and [Stear] for an overview of the latest developments.

It is easy to see that pattern containment defines a poset (partially ordered set) $\mathcal{P}$ on the set of all permutations of length $n$ for all $n>0$. This poset is the underlying object of all studies of pattern avoidance and containment. A classical question about any combinatorially defined poset is what its Möbius function is. A generalization of that question concerns the topology of the (order complexes of) intervals in $\mathcal{P}$, since the Möbius function of an interval $I=[a, b]$ in $\mathcal{P}$ equals the reduced Euler characteristic of the topological space determined by the order complex $\Delta(I)$, whose faces are the chains of the open interval $(a, b)$. In particular, we would like to know the homology and the homotopy type of intervals in $\mathcal{P}$.

The first results on the Möbius function of intervals of $\mathcal{P}$ were obtained by Sagan and Vatter [SV06], who used discrete Morse theory to compute the Möbius function for the poset of layered permutations; as they pointed out, this poset is easily seen to be isomorphic to a certain poset they studied of compositions of an integer. Later results about the Möbius function of $\mathcal{P}$ have been obtained by Steingrímsson and Tenner [ST10] and by Burstein et al. [BJJS11], the latter of which gave an effective formula for the Möbius function of intervals of separable permutations (those avoiding both of the patterns 2413 and 3142 ) and reduced the computation for decomposable permutations (those non-trivially expressible as direct sums) to that for indecomposable ones. Recently, Smith [Smi13] obtained the first systematic results for several classes of intervals of indecomposable permutations, including those intervals $[1, \pi]$ where $\pi$ is any permutation with exactly one descent.

In this extended abstract we present what seem to be the first major results on the topology of intervals $I$ in $\mathcal{P}$. (Although bounds on Betti numbers can be inferred from the results of Sagan and Vatter [SV06], they did not present any explicit such results.) As is conventional in topological combinatorics, we will say that $I$ has a property if the topological space determined by $\Delta(I)$ has that property. As is so often the case, our results on the topology of intervals are mostly based on showing that they are shellable. This implies that these intervals have the homotopy type of a wedge of spheres, where all the spheres are of the top dimension, that is, the same dimension as $\Delta(I)$, and the homology is thus only in the top dimension. In that case, the number of spheres equals, up to a sign depending only on rank, the Möbius function of the interval.

We first characterize those intervals that are disconnected, since an interval with a disconnected subinterval of rank at least 3 is certainly not shellable. An example of a disconnected interval is given in Figure 1.1. If a disconnected subinterval has rank at least 3 we qualify it as being non-trivial, since such a subinterval prevents an interval containing it from being shellable, as shown by Björner [Bjö80, Prop. 4.2]. (Note that an interval of rank 2 that is not a chain is disconnected, but shellable since its order complex is 0 -dimensional.) It turns out that "almost all" intervals in $\mathcal{P}$ have non-trivial disconnected subintervals and are thus not shellable. More precisely, given any permutation $\sigma$, the probability that the interval $[\sigma, \tau]$ has such a disconnected subinterval, for a randomly chosen permutation $\tau$ of length $n$, goes to one as $n$ 


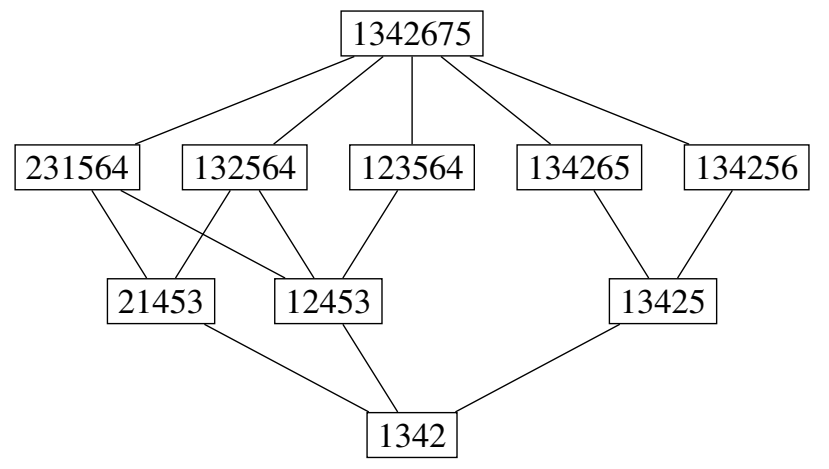

Fig. 1.1: The disconnected interval [1342, 1342675]

goes to infinity. Shellable intervals are thus, in this sense, an exception to the general rule. This seems to be just one manifestation of a more general property of $\mathcal{P}$ : it seems to be very hard to get a grip on its generic intervals. Even so, there are various substantial classes of intervals where results have been pried out in recent years, and almost certainly more is to come.

We give a very simple characterization of those intervals of layered permutations that are disconnected. This allows us to determine which intervals of layered permutations have no non-trivial disconnected subintervals and, in contrast to statements in the previous paragraph, we show that all such intervals are shellable. We conjecture that the same is true for intervals of separable permutations, that is, that the only obstruction to shellability of such an interval is a non-trivial disconnected subinterval.

We also present a unified (and simplified) version of the two fundamental propositions in [BJJS11. Propositions 1 and 2], which reduce the computation of the Möbius function for decomposable permutations to a computation involving their components.

The extended abstract is organized as follows. In Section 2 we collect some necessary definitions and observations. In Section 3 we show that almost all intervals in $\mathcal{P}$ are non-shellable. In Section 4 we show that disconnectivity is preserved under certain operations on intervals, and give conditions for these operations to actually give intervals isomorphic to the original ones. In Section 5 we give necessary and sufficient conditions for an interval of layered permutations to be disconnected, and show that having no non-trivial disconnected subintervals implies (and hence is equivalent to) shellability. In fact, our results here apply to a more general situation, namely to generalized subword order (see, for example, [MS12, SV06]) where the underlying poset is a rooted forest. In Section 6 we give a unified (and simplified) version of the two fundamental recursive formulas in [BJJS11] for the Möbius function of intervals $[\sigma, \tau]$ where $\tau$ is decomposable. Finally, in Section 7, we mention some open problems and questions. The full proofs of the results appearing here, and some further results, can be found in [MS].

\section{Preliminaries}

In this section, we establish terminology and notation that we will use repeatedly.

The letters of all our permutations $\pi$ are positive integers, and we use $|\pi|$ to denote the number of letters in $\pi$. As mentioned above, the definition of the partial order in the poset $\mathcal{P}$ refers only to the relative order of size of letters in permutations. Thus, deleting different letters from a given permutation can result in the 
same element of $\mathcal{P}$, such as when we delete either the 2 or the 3 from 416325 . The resulting permutations, 41635 and 41625 , are said to be order isomorphic, and they have the same standard form, namely 31524 , since 31524 is the (only) permutation of $\{1,2,3,4,5\}$ whose letters appear in the same order of size as in 41635 and 41625 . The map that takes a permutation to its standard form is referred to as flattening.

The direct sum of two permutations $\alpha$ and $\beta$, denoted $\alpha \oplus \beta$, is the concatenation of $\alpha$ and $\beta^{\prime}$, where $\beta^{\prime}$ is obtained from $\beta$ by adding to each of its letters the largest letter of $\alpha$. The skew sum of $\alpha$ and $\beta$, denoted $\alpha \ominus \beta$, is the concatenation of $\alpha^{\prime}$ and $\beta$, where $\alpha^{\prime}$ is obtained from $\alpha$ by adding to each of its letters the largest letter of $\beta$. In particular, if $\alpha$ and $\beta$ are in standard form, then so are $\alpha \oplus \beta$ and $\alpha \ominus \beta$. For example, if $\alpha=213$ and $\beta=3142$, then $\alpha \oplus \beta=2136475$ and $\alpha \ominus \beta=6573142$. We say that a permutation is decomposable (respectively skew decomposable) if it is the direct sum (resp. skew sum) of two nonempty permutations, otherwise it is indecomposable (resp. skew indecomposable). Clearly, every permutation has a unique finest decomposition, that is, a decomposition into the maximum number of indecomposable components. Note that a permutation cannot be both decomposable and skew decomposable, so every permutation is either indecomposable or skew indecomposable (or both).

As always in posets, the closed interval $[\sigma, \tau]$ in $\mathcal{P}$ is the set $\{\pi \mid \sigma \leq \pi \leq \tau\}$, and the open interval $(\sigma, \tau)$ (the interior of $[\sigma, \tau])$ is the set $\{\pi \mid \sigma<\pi<\tau\}$, where " $<$ " and " $\leq$ " have the usual meaning. When we talk about topological properties of an interval $I=[\sigma, \tau]$ such as connectedness and shellability (to be discussed later), the interval inherits these properties from the topological space determined by the order complex of the open interval $(\sigma, \tau)$, that is, from the simplicial complex whose faces are the chains of $(\sigma, \tau)$. We denote this order complex by $\Delta(I)$ or by $\Delta(\sigma, \tau)$. The rank of a closed interval $[\sigma, \tau]$ is one less than the maximum possible number of elements in a chain $\sigma<\pi_{1}<\pi_{2}<\cdots<\pi_{k}<\tau$ and the rank of an element $\pi \in[\sigma, \tau]$ is the rank of $[\sigma, \pi]$. When we talk about the rank of a subinterval $\left[\sigma^{\prime}, \tau^{\prime}\right]$ or $\left(\sigma^{\prime}, \tau^{\prime}\right)$, we always mean the rank of the closed interval, although topological properties of such an interval are determined by $(\sigma, \tau)$, as mentioned above.

We do not state the definition of shellability here since we have already stated the facts we need about shellability: that shellability of an interval completely determines its topology (a wedge of spheres of the top dimension), that disconnected intervals of rank at least 3 are not shellable, and that an interval of a poset is not shellable if it contains a subinterval that is not shellable. Our technique for showing shellability will be CL-shellability [BW82, BW83]. For background on these concepts we refer the reader to [Wac07.

\section{Almost all intervals are non-shellable}

In studying examples of intervals in $\mathcal{P}$, one quickly realizes that their structure is certainly not simple in general. One cause for this is stated in the title of this section and is made precise by the results below. We begin with a preliminary lemma which also has implications in later sections.

Lemma 3.1 Let $\sigma$ be a permutation of length $k \geq 2$. If $\sigma$ is indecomposable then the open interval $(\sigma, \sigma \oplus \sigma)$ is disconnected. Otherwise, if $\sigma$ is skew indecomposable, the open interval $(\sigma, \sigma \ominus \sigma)$ is disconnected.

Since every permutation is either indecomposable or skew indecomposable (or both), Lemma 3.1 can be applied in the proof of the next result. 
Theorem 3.2 Given a permutation $\sigma$, let $P(n)$ be the probability that the interval $[\sigma, \tau]$ has a non-trivial disconnected subinterval, where $\tau$ is a randomly chosen permutation of length $n$. Then

$$
\lim _{n \rightarrow \infty} P(n)=1 .
$$

Thus almost all intervals $[\sigma, \tau]$ in $\mathcal{P}$ are not shellable.

\section{Disconnectivity of intervals}

Clear examples of non-shellable intervals $[\sigma, \tau]$ are those for which $(\sigma, \tau)$ is disconnected with $|\tau|-|\sigma| \geq$ 3. See Figures 1.1 and 4.1 for such examples. In fact, for intervals of rank exactly 3 , shellability of $(\sigma, \tau)$ is equivalent to connectivity since $\Delta(\sigma, \tau)$ is just a graph, that is, one-dimensional. Moreover, if an interval contains a subinterval that is not shellable, then it is itself not shellable [Bjö80. Prop. 4.2], as mentioned above, leading to further relevance of disconnected intervals. For example, the open interval $(123,1342675)$ is connected, but its open subinterval $(1342,1342675)$ is not, and thus $(123,1342675)$ is not shellable. In fact, "most" non-shellable intervals violate shellability because they contain a nontrivial disconnected subinterval, an assertion made precise by Theorem 3.2 Also compare Theorem 5.4 . which shows that, in the case of layered permutations, any non-shellable interval contains a non-trivial disconnected subinterval. In summary, if we are to study shellability in the permutation pattern poset, the study of disconnectivity is a natural place to start.

The first examples of disconnected intervals $(\sigma, \tau)$ with $|\tau|-|\sigma| \geq 3$ occur when $|\tau|=6$. One such example is shown in Figure 4.1 and others follow from Lemma 3.1. such as [321,321 $\oplus$ 321].

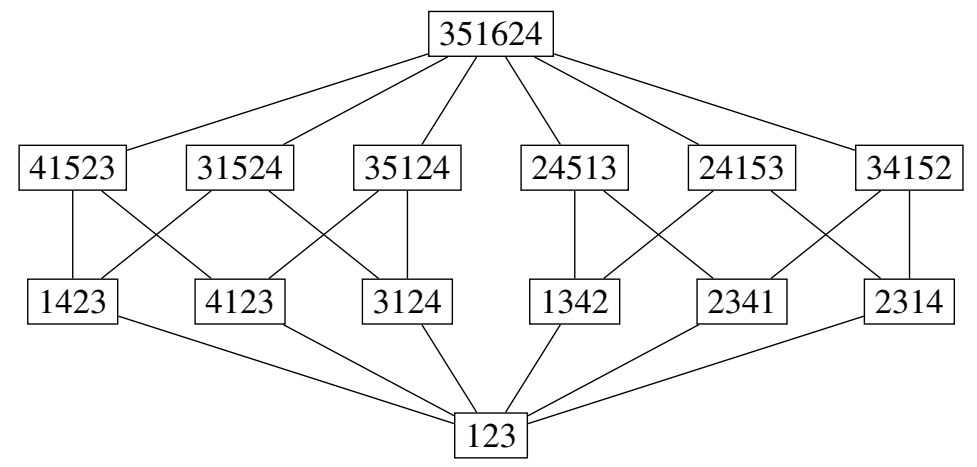

Fig. 4.1: The interval [123, 351624]

\subsection{Preservation of disconnectivity under augmentation}

In practice, many disconnected intervals are of the form $(\alpha \oplus \sigma, \alpha \oplus \tau)$ for some disconnected interval $(\sigma, \tau)$. Our next result explains this phenomenon. Its proof, which is certainly not trivial, relies on a classification of disconnected intervals in $\mathcal{P}$; this classification is omitted from this extended abstract but can be found in $[\mathrm{MS}]$. 
Theorem 4.1 Suppose $(\sigma, \tau)$ is a disconnected interval. Then for any permutation $\alpha$, the open interval $(\alpha \oplus \sigma, \alpha \oplus \tau)$ is also disconnected.

Applying certain involutions to permutations, we can show the following variants of Theorem 4.1

Corollary 4.2 Suppose $(\sigma, \tau)$ is a disconnected interval. Then for any permutation $\alpha$, all of the following augmentations of $(\sigma, \tau)$ are also disconnected:

$$
(\alpha \oplus \sigma, \alpha \oplus \tau) ; \quad(\alpha \ominus \sigma, \alpha \ominus \tau) ; \quad(\sigma \oplus \alpha, \tau \oplus \alpha) ; \quad(\sigma \ominus \alpha, \tau \ominus \alpha) .
$$

Consequently, any sequence of augmentations from these four types preserves disconnectivity.

Combined with Lemma 3.1 for example, Corollary 4.2 allows us to easily generate infinite classes of disconnected intervals.

Our "augmentation" terminology does not mean to suggest that the intervals themselves are larger, just that the top and bottom elements of the corresponding closed intervals are longer.

\subsection{Isomorphism under augmentation}

While Corollary 4.2 shows that disconnectivity is preserved under augmentation, under certain conditions we actually get an isomorphism, as we now show.

Theorem 4.3 Consider an interval $[\sigma, \tau]$ and let $\alpha$ and $\gamma$ be indecomposable permutations and $\beta$ and $\delta$ be skew indecomposable permutations.

a. If $\alpha \oplus \sigma \not \leq \tau$, then $[\sigma, \tau] \cong[\alpha \oplus \sigma, \alpha \oplus \tau]$.

b. If $\beta \ominus \sigma \not \leq \tau$, then $[\sigma, \tau] \cong[\beta \ominus \sigma, \beta \ominus \tau]$.

c. If $\sigma \oplus \gamma \not \leq \tau$, then $[\sigma, \tau] \cong[\sigma \oplus \gamma, \tau \oplus \gamma]$.

d. If $\sigma \ominus \delta \not \leq \tau$, then $[\sigma, \tau] \cong[\sigma \ominus \delta, \tau \ominus \delta]$.

In words, each part says that the interval $[\sigma, \tau]$ is isomorphic to its augmentation when the augmented interval does not intersect $[\sigma, \tau]$. For example, referring to Figure 4.1, since $1 \oplus 123=1234 \not \leq 351624$, we get $[123,351624] \cong[1234,1462735]$. In fact, the isomorphism simply sends $\pi$ to $1 \oplus \pi$.

As in Corollary 4.2. a sequence of the augmentations from Theorem 4.3 preserves isomorphism as long as the relevant conditions are satisfied. For example, for $\alpha$ and $\gamma$ indecomposable, we get that $[\sigma, \tau] \cong[\alpha \oplus \sigma \oplus \gamma, \alpha \oplus \tau \oplus \gamma]$ as long as $\alpha \oplus \sigma \not \leq \tau$ and $\alpha \oplus \sigma \oplus \gamma \not \leq \alpha \oplus \tau$, with the latter condition being equivalent to $\sigma \oplus \gamma \not \leq \tau$. For example,

$$
[321,321 \oplus 321] \cong[312 \oplus 321 \oplus 231,312 \oplus 321 \oplus 321 \oplus 231] .
$$

Theorem 4.3 a) does not identify all isomorphisms of the form $[\sigma, \tau] \cong[\alpha \oplus \sigma, \alpha \oplus \tau]$. As a basic example, we have $[1,12] \cong[12,123]$. The same is true even if we restrict to disconnected intervals, with $[1324,1365724] \cong[1 \oplus 1324,1 \oplus 1365724]$ serving as an example. Looking at this latter isomorphism, one might wonder if it is often the case that

$$
\left[1^{r} \oplus \sigma, 1^{r} \oplus \tau\right] \cong\left[1^{r+1} \oplus \sigma, 1^{r+1} \oplus \tau\right]
$$

for sufficiently large $r$, where $1^{r}$ denotes $1 \oplus 1 \oplus \cdots \oplus 1$ with $r$ copies of 1 . The next result shows that intervals $\left[1^{r} \oplus \sigma, 1^{r} \oplus \tau\right]$ eventually stabilize as $r$ increases. 
Proposition 4.4 For any interval $[\sigma, \tau]$, we have

$$
\left[1^{r} \oplus \sigma, 1^{r} \oplus \tau\right] \cong\left[1^{r+1} \oplus \sigma, 1^{r+1} \oplus \tau\right]
$$

whenever $r \geq|\tau|-|\sigma|-1$. In fact, if $\tau$ takes the form $1^{s} \oplus \tau^{\prime}$ for some $\tau^{\prime}$, then (4.1) holds whenever $r \geq|\tau|-|\sigma|-s-1$.

The bound on $r$ in Proposition 4.4 is sharp in the sense that there exist cases where $\left[1^{r} \oplus \sigma, 1^{r} \oplus \tau\right]$ and $\left[1^{r+1} \oplus \sigma, 1^{r+1} \oplus \tau\right]$ are not isomorphic when $r=|\tau|-|\sigma|-s-2$. One example with $s=0$ is given by $[\sigma, \tau]=[132,213465]$ since

$$
[1 \oplus 132,1 \oplus 213465] \approx[1 \oplus 1 \oplus 132,1 \oplus 1 \oplus 213465] .
$$

\section{Layered permutations and generalized subword order}

The goal of this section is to completely determine disconnectivity and shellability conditions for intervals of layered permutations. In contrast with Theorem 3.2, we will give an infinite class of intervals that are shellable. In fact, our technique will carry through to the more general case of intervals $[u, w]$ in generalized subword order when the ordering on the alphabet $P$ consists of a rooted forest. We begin with the necessary preliminaries.

Definition 5.1 A permutation is said to be layered if the letters of each component of its finest decomposition are decreasing.

For example, $32165798=321 \oplus 21 \oplus 1 \oplus 21$ is layered. We see that every layered permutation is uniquely determined by its composition of layer lengths; it will be helpful for think of layered permutations in terms of these compositions.

To put these compositions in a more general setting, let $P$ be a poset and let $P^{*}$ denote the set of finite words in the alphabet consisting of the elements of $P$. We define generalized subword order on $P^{*}$ as follows.

Definition 5.2 Let $P$ be a poset. For $u, w \in P^{*}$, we write $u \leq w$ and say that $u$ is less than or equal to $w$ in generalized subword order if there exists a subword $w\left(i_{1}\right) w\left(i_{2}\right) \cdots w\left(i_{k}\right)$ of the same length as $u$ such that

$$
u(j) \leq_{P} w\left(i_{j}\right) \text { for all } j \text { with } 1 \leq j \leq k .
$$

Note that we compare $u(j)$ and $w\left(i_{j}\right)$ in the inequality above according to the partial order $P$. For example, if $P$ is an antichain, then generalized subword order on $P^{*}$ is equivalent to ordinary subword order. More importantly for us, if $P$ is the usual order $\mathbb{P}$ on the positive integers, then generalized subword order amounts to pattern containment order on layered permutations. For example, with $P=\mathbb{P}$, that $112 \leq 3212$ in generalized subword order is equivalent to the inequality $1 \oplus 1 \oplus 21 \leq 321 \oplus 21 \oplus 1 \oplus 21$ for layered permutations, i.e., $1243 \leq 32165798$.

We will work in the language of generalized subword order throughout the remainder of this section, referring to layered permutations, or equivalently to the $P=\mathbb{P}$ case, from time to time. Let us introduce some new notation and translate some of our previous notation and terminology to this generalized subword setting. We will use $P$ throughout to denote our ordered alphabet, and let $P_{0}$ denote $P$ with a bottom element 0 adjoined. We will typically use $u$ and $w$ in place of $\sigma$ and $\tau$, and $|w|$ will denote the rank of $w$ in 
$P^{*}$, which is equal to the sum of the ranks of the letters of $w$ in $P_{0}$. For example, with $P=\mathbb{P},|3212|=8$, which is consistent with the notation $|32154687|=8$ for the corresponding layered permutation. Ranks are defined in the usual way in $P_{0}$ since we will hereafter restrict to the case where $P$ is a rooted forest, meaning that it consists of a disjoint union of trees, each rooted at a unique bottom element. Equivalently, every element of $P_{0}$ except 0 covers exactly one element. Note that $P$ being a rooted forest includes the cases when $P$ is an antichain or a chain.

Theorem 5.3 Let $P$ be a rooted forest, and let $u, w \in P^{*}$ with $|w|-|u| \geq 3$. Then $(u, w)$ is disconnected if and only if $u$ and $w$ are the concatenations $u=\left(v_{1}, a, v_{2}\right)$ and $w=\left(v_{1}, a, a, v_{2}\right)$ for some letter $a \in P$ and for $v_{1}, v_{2} \in P^{*}$.

Note that this result implies that for an interval $[\sigma, \tau]$ of layered permutations with $|\tau|-|\sigma| \geq 3$ to be disconnected, the composition of $\sigma$ is obtained from the composition of $\tau$ by deleting a component that has size at least 3 and that is equal to its preceding component in $\tau$. An example of this is [215436, 215438769], with corresponding compositions 231 and 2331, respectively.

As in Theorem 3.2. we know that if an interval contains a non-trivial disconnected subinterval, then it is not shellable. It is natural to ask which intervals $[u, w]$ in $P^{*}$ without such disconnected subintervals are shellable. Our second main result of this section tells us that when $P$ is a rooted forest, all such intervals are shellable. This result is a companion to a result from [MS12], which states that if $P_{0}$ is finite and has rank at most 2 , then any interval in $P^{*}$ is shellable.

We will prove shellability using the notion of CL-shellability, introduced by Björner and Wachs [BW82], where it is called "L-shellability" and where chains are read from top to bottom. We will follow what is now the customary definition of CL-shellability from [BW83], where chains are instead read from bottom to top. Because our chain labeling will be read from top to bottom, we will actually show that the dual of the interval $[u, w]$ is CL-shellable and hence shellable; this implies the shellability of $[u, w]$ since the order complex of $[u, w]$ is clearly isomorphic to that of its dual. In this case, we say that $[u, w]$ is dual $C L$-shellable. Readers interested in an exposition of CL-shellability (and a wealth of other information about poset topology) are referred to [Wac07].

Theorem 5.4 Let $P$ be a rooted forest. Suppose an interval $[u, w]$ in $P^{*}$ does not contain a non-trivial disconnected subinterval. Then $[u, w]$ is dual CL-shellable.

For example, with $P=\mathbb{P}$, we can use Theorem 5.3 to check that $[131,33121]$ has no disconnected subintervals, and so it is dual CL-shellable.

It will be helpful to introduce and give relevant terminology for the chain labeling we use since there is some trickery involved. A natural chain labeling of $[u, w]$ would label the edge $v^{\prime} \rightarrow v$ along $C$ by the position in $w$ that is decreased. If there is a choice of positions that will both yield $v$, then we choose the smaller label. For example, with $P=\mathbb{P}$ and using 0 as a placeholder for a deleted letter,

$$
3212 \stackrel{1}{\longrightarrow} 2212 \stackrel{2}{\longrightarrow} 2112 \stackrel{2}{\longrightarrow} 2012 \stackrel{1}{\longrightarrow} 1012 .
$$

This is exactly the labeling used in [SV06], and we will call it the position labeling. Unfortunately, this labeling is too simple to give a CL-labeling, as illustrated by Figure 5.1 a) for the case $P=\mathbb{P}$, where all three maximal chains are weakly increasing from top to bottom. To rectify this situation, we make the following special modification to the position labeling. Suppose $w \rightarrow v \rightarrow u$ and $w$ has a consecutive sequence of $b$ 's that is maximal under inclusion, where $b$ is an element of rank 2 in $P_{0}$. Since $P$ is a rooted 


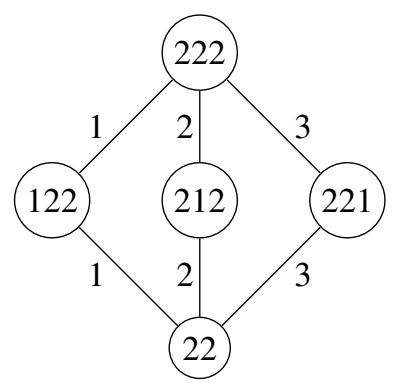

(a)

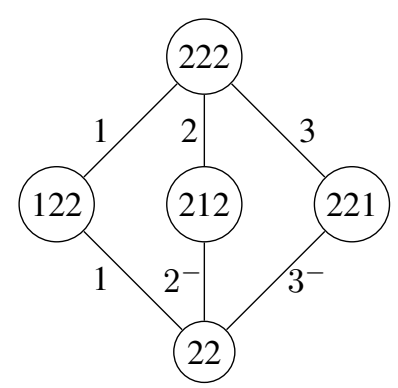

(b)

Fig. 5.1: Labeling according to the position decreased as in (a) can result in more than one increasing chain. In this case, we modify the labels so that only the chain that deletes the leftmost 2 has increasing labels, as in (b).

forest, $b$ covers a unique element $a$ in $P$, and $a$ is a minimal element of $P$. Suppose that the $i$-th of these $b$ 's in the consecutive sequence in $w$ is decreased to $a$ in going to $v$ and then that $a$ is deleted in going to $u$. If $i>1$, then change the label $k$ on $v \rightarrow u$ to $k^{-}$, where $k-1<k^{-}<k$ (if we prefer to be specific, $k^{-}=k-0.5$ will certainly suffice). The result is that only the chain that deletes the leftmost $b$ in the consecutive sequence gets weakly increasing labels from top to bottom in $[u, w]$. An example of this modified labeling in the case $P=\mathbb{P}$ is shown in Figure 5.1(b). While this modification may seem somewhat arbitrary, it is exactly what we need to get a dual CL-labeling. We will call the labeling just described the modified position labeling.

As a consequence of shellability, for $P$ a rooted forest, we get that any interval $[u, w] \in P^{*}$ that does not contain a non-trivial disconnected subinterval is homotopic to a wedge of $|\mu(u, w)|$ spheres, each of the top dimension $|w|-|u|-2$. Therefore, we know the homotopy type completely since a formula for $\mu(u, w)$ is given in [SV06]. A formula for $\mu(u, w)$ for general $P$ is the main result of [MS12]. Modifying this latter formula for the case of decomposable permutations is the subject of the next section.

\section{The Möbius function of decomposable intervals}

Suppose $\tau$ is a decomposable permutation and let $\tau=\tau_{1} \oplus \cdots \oplus \tau_{t}$ be its finest decomposition throughout this section, referring to the $\tau_{i}$ as the components of $\tau$. Results in [BJJS11, Prop. 1 and 2] give recurrences that reduce the computation of the Möbius function $\mu(\sigma, \tau)$ to Möbius function calculations of the form $\mu\left(\sigma^{\prime}, \tau^{\prime}\right)$ where $\tau^{\prime}$ is a single component of $\tau$ and $\sigma^{\prime}$ is a direct sum of consecutive components of $\sigma$. For example, a corollary of these results of [BJJS11] is that if $\sigma$ is indecomposable, then $\mu(\sigma, \tau)$ is either 0 or $\pm \mu\left(\sigma, \tau_{1}\right)$, depending on the form of $\tau$.

A disadvantage of the results of [BJJS11] is that the recurrences are given in the form of two different propositions, one for the case $\tau_{1}=1$ and one for $\tau_{1}>1$; the formulas for $\mu(\sigma, \tau)$ in the two propositions look very different and neither is simple. We now state our new formula, which replaces the two propositions by a single recursive expression for $\mu(\sigma, \tau)$.

Theorem 6.1 Consider permutations $\sigma$ and $\tau$ and let $\tau=\tau_{1} \oplus \cdots \oplus \tau_{t}$ be the finest decomposition of $\tau$. 
Then

$$
\mu(\sigma, \tau)=\sum_{\sigma=\varsigma_{1} \oplus \ldots \oplus \varsigma_{t}} \prod_{1 \leq m \leq t} \begin{cases}\mu\left(\varsigma_{m}, \tau_{m}\right)+1 & \text { if } \varsigma_{m}=\emptyset \text { and } \tau_{m-1}=\tau_{m} \\ \mu\left(\varsigma_{m}, \tau_{m}\right) & \text { otherwise }\end{cases}
$$

where the sum is over all direct sums $\sigma=\varsigma_{1} \oplus \ldots \oplus \varsigma_{t}$ such that $\emptyset \leq \varsigma_{m} \leq \tau_{m}$ for all $1 \leq m \leq t$.

The condition $\tau_{m-1}=\tau_{m}$ is considered false when $m=1$ since $\tau_{0}$ does not exist. Theorem 6.1 is inspired by, and is an exact analogue of, the formula from [MS12] for the Möbius function for generalized subword order. Unfortunately, we have not been able to find a way to obtain Theorem 6.1 as an application of the formula for generalized subword order. Instead, our proof of Theorem 6.1 works by showing that it gives the same recursive expressions for $\mu(\sigma, \tau)$ as the propositions of [BJJS11].

Example 6.2 To see Theorem 6.1 in action, let us compute $\mu(12,24136857)=\mu(12,2413 \oplus 2413)$. It is straightforward to compute by hand that $\mu(12,2413)=3$ and $\mu(1,2413)=-3$. We also know that $\mu(\emptyset, \tau)=0$ for any $\tau>1$. On the other hand, $[12,2413 \oplus 2413]$ has 62 elements and 223 edges, meaning that computing $\mu(12,24136857)$ directly is a much less pleasant exercise. Instead, applying Theorem 6.1, there are three terms in the sum:

○ $12=1 \oplus 1$ contributes $\mu(1,2413) \mu(1,2413)=9$;

○ $12=\emptyset \oplus 12$ contributes $\mu(\emptyset, 2413) \mu(12,2413)=0$;

$\circ 12=12 \oplus \emptyset$ contributes $\mu(12,2413)(\mu(\emptyset, 2413)+1)=3$, with the +1 arising because we have $\varsigma_{2}=\emptyset$ and $\tau_{1}=\tau_{2}$.

Therefore $\mu(12,24136857)=12$.

\section{Open problems}

\subsection{Non-shellable intervals without disconnected subintervals}

In view of Theorem 5.4 it is natural to ask if there exist intervals $[\sigma, \tau]$ that are not shellable but have no non-trivial disconnected subintervals. While we do not have a good way to test shellability computationally, we can test whether a poset is Cohen-Macaulay, i.e., whether all the homology is in the top dimension, which is implied by shellability. The first intervals $[\sigma, \tau]$ that have no non-trivial disconnected subintervals but are not Cohen-Macaulay, and thus not shellable, occur when $|\tau|=7$. One such example is $[123,3416725]$. It would be interesting to determine if there is something simple about the structure of such intervals that implies their non-shellability.

\subsection{Separable permutations}

By Theorem 5.4, we know that an interval of layered permutations of rank at least 3 is shellable if and only if it does not contain any non-trivial disconnected subintervals. Does the same property hold for any larger class of intervals? It does not hold in general for $[\sigma, \tau]$, and not even with $\sigma$ and $\tau$ decomposable, since $[1 \oplus 123,1 \oplus 3416725]$ is not shellable but has no non-trivial disconnected subintervals. Moreover, the interval $[1 \oplus 123,1 \oplus 3416725]$ is not isomorphic to [123,3416725], so the non-shellability of the former interval is not a trivial consequence of the non-shellability of the latter one (where 3416725 is indecomposable). 
Layered permutations are special cases of separable permutations. A permutation is separable if it can be generated from the permutation 1 by successive sums and skew sums. In other words, a permutation is separable if it is equal to 1 or can be expressed as the sum or skew sum of separable permutations. For example, $52143=1 \ominus((1 \ominus 1) \oplus(1 \ominus 1))$. Equivalently, a permutation is separable if it avoids the patterns 2413 and 3142 (see [BBL98]). Consequently, if $\tau$ is separable, then any $\sigma \leq \tau$ is also separable.

Conjecture 7.1 An interval $[\sigma, \tau]$ of separable permutations with $|\tau|-|\sigma| \geq 3$ is shellable if and only if it has no non-trivial disconnected subintervals.

It was shown in [BJJS11, Cor. 24 and 25] that for a separable permutation $\tau$, the Möbius function $\mu(1, \tau)$ can only take the values 0,1 and -1 , and that the same is true of $\mu(\sigma, \tau)$ if $\sigma$ occurs precisely once in $\tau$. If true, Conjecture 7.1 would therefore imply that, for such $\sigma$ and $\tau$, intervals $[1, \tau]$ and $[\sigma, \tau]$ are each either contractible or homotopy equivalent to a single sphere (of dimension $|\tau|-3$ and $|\tau|-|\sigma|-2$, respectively).

As discussed at the start of Section 4, the "only if" direction of Conjecture 7.1 is known and the "if" direction holds for $[\sigma, \tau]$ of rank 3. As other evidence in favor of the "if" direction, we have found, by computer tests, that all such intervals with $|\tau| \leq 9$ are Cohen-Macaulay. A weaker condition than $[\sigma, \tau]$ being Cohen-Macaulay is that the Möbius function alternates in sign, i.e., the sign of every subinterval of $[\sigma, \tau]$ is $(-1)^{r}$ where $r$ is the rank of the subinterval [Sta86, Sta12, Prop. 3.8.11]. We have checked that if $[\sigma, \tau]$ has no non-trivial disconnected subintervals, then the Möbius function of $[\sigma, \tau]$ alternates in sign whenever $|\tau| \leq 10$ and also for $|\sigma|=7$ when $|\tau|=11$.

In an attempt to extend the proof of Theorem 5.4 to separable permutations, one might first try to extend the "if" direction of Theorem 5.3. Such an extension does exist, and follows from Lemma 3.1 and Corollary 4.2

Lemma 7.2 Let $\sigma$ and $\tau$ be separable permutations with $|\tau|-|\sigma| \geq 3$. Suppose $\tau$ has a contiguous subword of contiguous letters that, after flattening, takes the form $\pi \oplus \pi$ with $\pi$ indecomposable or $\pi \ominus \pi$ with $\pi$ skew indecomposable. Suppose $\sigma$ is obtained from $\tau$ by removing one of these copies of $\pi$. Then $(\sigma, \tau)$ is disconnected.

We can also ask if the converse of Lemma 7.2 is also true, although in the layered case the corresponding statement was not needed in the proof of Theorem 5.4

\section{References}

[BBL98] Prosenjit Bose, Jonathan F. Buss, and Anna Lubiw. Pattern matching for permutations. Inform. Process. Lett., 65(5):277-283, 1998.

[BJJS11] Alexander Burstein, Vít Jelínek, Eva Jelínková, and Einar Steingrímsson. The Möbius function of separable and decomposable permutations. J. Combin. Theory Ser. A, 118(8):2346-2364, 2011.

[Bjö80] Anders Björner. Shellable and Cohen-Macaulay partially ordered sets. Trans. Amer. Math. Soc., 260(1):159-183, 1980.

[BW82] Anders Björner and Michelle Wachs. Bruhat order of Coxeter groups and shellability. Adv. in Math., 43(1):87-100, 1982. 
[BW83] Anders Björner and Michelle Wachs. On lexicographically shellable posets. Trans. Amer. Math. Soc., 277(1):323-341, 1983.

[Kit11] Sergey Kitaev. Patterns in Permutations and Words. Monographs in Theoretical Computer Science. Springer-Verlag, 2011.

[Knu75] Donald E. Knuth. The art of computer programming. Addison-Wesley Publishing Co., Reading, Mass.-London-Amsterdam, second edition, 1975. Volume 1: Fundamental algorithms, Addison-Wesley Series in Computer Science and Information Processing.

[MS] Peter R. W. McNamara and Einar Steingrímsson. On the topology of the permutation pattern poset. Preprint. arXiv:1305.5569.

[MS12] Peter R. W. McNamara and Bruce E. Sagan. The Möbius function of generalized subword order. Adv. Math., 229(5):2741-2766, 2012.

[Rog78] D. G. Rogers. Ascending sequences in permutations. Discrete Math., 22(1):35-40, 1978.

[Smi13] Jason P. Smith. On the Möbius function for permutations with one descent. Preprint. arXiv:1306.5926, 2013.

[SS85] Rodica Simion and Frank W. Schmidt. Restricted permutations. European J. Combin., 6(4):383-406, 1985.

[ST10] Einar Steingrímsson and Bridget Eileen Tenner. The Möbius function of the permutation pattern poset. J. Comb., 1(1, [ISSN 1097-959X on cover]):39-52, 2010.

[Sta86] Richard P. Stanley. Enumerative combinatorics. Vol. I. The Wadsworth \& Brooks/Cole Mathematics Series. Wadsworth \& Brooks/Cole Advanced Books \& Software, Monterey, CA, 1986. With a foreword by Gian-Carlo Rota. Second printing, Cambridge University Press, Cambridge/New York, 1997.

[Sta12] Richard P. Stanley. Enumerative combinatorics. Volume 1, volume 49 of Cambridge Studies in Advanced Mathematics. Cambridge University Press, Cambridge, second edition, 2012.

[Stear] Einar Steingrímsson. Some open problems on permutation patterns. Preprint. $\operatorname{arXiv}: 1210.7320$, London Math. Soc. Lecture Note Ser., to appear.

[SV06] Bruce E. Sagan and Vincent Vatter. The Möbius function of a composition poset. J. Algebraic Combin., 24(2):117-136, 2006.

[Wac07] Michelle L. Wachs. Poset topology: tools and applications. In Geometric combinatorics, volume 13 of IAS/Park City Math. Ser., pages 497-615. Amer. Math. Soc., Providence, RI, 2007. 